BULL. AUSTRAL. MATH. SOC.

VOL. $24(1981), 405-431$.

\title{
MANUFACTURING SEQUENCES FOR \\ THE ECONOMIC LOT SCHEDULING PROBLEM
}

\author{
James M. Fitz-Gerald and Elizabeth J. Billington
}

\begin{abstract}
In the basic Economic Lot Scheduling problem, a production schedule is required to manufacture sequentially a number of products on a single machine, with the schedule chosen to minimize set-up and inventory costs. The products suffer continuous demand, and no shortfall is allowed. A recent approach involves repetitions of a production cycle (such as $A B C B C$ for three products $A, B$ and $C$, with manufacturing times chosen to prevent shortage occurring); an exhaustive search is performed over a large set of possible cycles to discover the optimal schedule.

This paper discusses the question "How many such cycles need be examined?", since the answer is very relevant to practical application of the method. The case of three products is considered. Complete information is obtained for cycles up to length 12 (that is, 12 production switch overs), and partial results for longer ones. An estimate, apparently reasonable, is obtained for cycles of any length. The major trend to emerge is that surprisingly few cycles are involved.
\end{abstract}

\section{Introduction}

In the simplest form of the Economic Lot Scheduling problem, a single machine is used to manufacture a number of different products, at known, and in general different, rates of production. Each product has a

Received 25 May 1981. 
continuous, steady demand. Only one product can be manufactured at a time, so that during the time in which product $A$ is being produced, enough inventory must be accumulated (production must obviously exceed demand) to meet demand during the time interval until the next production run of $A$. Machine capacity is sufficient to cope with demand for all products, and there may in fact be slack time.

Each time that a change-over occurs, a set-up cost is incurred, suggesting that long production runs are preferable. On the other hand, storage costs are incurred for holding inventory that are proportional to the level of stock and the time for which it is held. This suggests that short production runs are more economical. The Economic Lot Scheduling problem is to determine the sequencing of manufacture in order to minimize total costs.

It is well known that for a single product the optimal schedule is to produce cyclically, alternately accumulating and consuming, with a constant period determined by the "square root law" from the set-up and holding costs. In theory, the optimal multi-product solution would be a superposition of the independent "best" cycles; in practice, unless some remarkable coincidences exist, overlapping of the production runs will occur, rendering the scheme infeasible. However, the independent-cycle solution provides a lower cost bound on the optimal feasible solution.

The simplest feasible solution is the common cycle schedule. Products are made in strict rotation, with a common period. Individual manufacturing times are selected to cope with demands, and the period is chosen to minimize costs over this very restricted class of solutions. It is not difficult to show that the existence of a common cycle solution guarantees sufficient machine capacity and vice versa. Clearly, the best common cycle solution provides an upper cost bound on the optimal feasible solution.

Better feasible solutions have been sought for over thirty years. Virtually all published work has used the "basic period" approach or its extensions. This class of solutions requires that each product has a strictly periodic schedule, with equally-spaced manufacturing periods of equal length, but that each product may have its own period. All such periods must, however, be integral multiples of a basic period, to avoid infeasibility due to overlap. The hope is that each period may be made reasonably close to the independent "best" value. What is found, if such a 
solution is available (and discoverable!) is an overall cycle, of period equal to the least common multiple of the individual periods, in which each product appears at least once.

Considerable ingenuity is required to find multipliers which allow the manufacturing intervals to be "fitted-in" to the schedule. Almost invariably, some version of a dynamic programme is used, as for example in Bomberger [1]. Decision rules, often heuristic, provide for cost minimization over the limited family of schedules found. It is clear that these methods optimize over a restricted class of feasible solutions, and that the restrictions become severe as the machine approaches its capacity limit, when little slack time is available to allow fitting-in adjustments. Furthermore, the computing requirements of such a search-and-modify technique are likely to be high. A review of work along these lines has been given by Elmaghraby [2].

Recently Fitz-Gerald [3] introduced the concept of the repetitive multiple period cycle, most easily explained in terms of an example. Suppose three products $A, B$ and $C$ are to be manufactured. A cycle is any sequence in which each of $A, B$ and $C$ appears at least once, and no immediate repetitions occur. Thus $A B C, A B C B C, A C A B C B$ are all cycles, while $B C B C$ and $A B B A C$ are not. A cycle is repetitively feasible for a cycle time $T$ if, by manufacturing in the order specified, it is possible to satisfy product demands for the period $T$, without leaving shortages or requiring overlaps of production, and without requiring production for longer than $T$. The cycle is thus self-contained, and a schedule consisting of repetitions of the cycle provides a feasible solution to the Economic Lot Scheduling problem. Such a schedule is called a repetitive multiple period cycle solution. The "multiple period" refers to the fact that each appearance of a product in the cycle will involve a manufacturing time different from those for other appearances of that product. There will be periodic behaviour associated with each appearance, all of period $T$, and hence multiply-periodic behaviour for the collection of all appearances of any product.

Fitz-Gerald [3] has shown that if the common cycle solution is feasible, all possible cycles are repetitively feasible. If set-up is instantaneous, any cycle time may be used; if set-up time is required, a lower limit is placed on the range of available cycle times for a given 
cycle. If slack time is available, and a basic-period type solution exists, such a solution will be included among the repetitive multiple period cycle solutions (and will represent the optimal distribution of slack time for that cycle). Clearly, for a given number of products, all cycles may be listed, with an epnumeration procedure based on cycle complexity.

The modus operandi for a practical problem is to evaluate systematically the optimal cost per unit time for all cycles in the list. For the case of no slack time, each evaluation requires solution of a set of linear equations for the manufacturing times, and substitution into a simple formula. The order of the matrix to be inverted is the length of the cycle (for example, 5 for the cycle $A B C B C$ ). No search procedure is needed. In practice, evaluation will be terminated when cycles of impractical complexity are reached, or when the lower bound has been sufficiently closely approximated. Some modification of the evaluation process is needed to make efficient use of any available slack time.

The repetitive multiple period cycle approach provides access to a very large class of solutions to the Economic Lot Scheduling problem. Its utility is limited only by the computational effort involved, and hence by the number of evaluations needed as the cycle length increases. This paper examines in some detail the problem of the number of cycles for the threeproduct case. Complete results are obtained for cycles up to length 12 , together with partial information and an estimate for more complex cycles.

\section{Framework, definitions and notation}

\subsection{PRELIMINARY CONCEPTS}

The length of a cycle, $P$, is the number of set-ups or places in that cycle; for example, $A B C B A C$ has length 6 . The repetition number $n_{A}$ is the number of places occupied by $A$; in the example, $n_{A}=2=n_{B}=n_{C}$. A composition is an ordered set of repetition numbers, $\left(n_{A}, n_{B}, n_{C}\right)$ when there are three products. A standard composition is one in which the elements form a non-decreasing sequence; for example, $(2,3,5)$ is a standard composition with $n_{A}=2, n_{B}=3$, and $n_{C}=5$. 


\subsection{MODES}

Consider a cycle $A B A B C B$, constructed from the composition $(2,3,1)$. This may be obtained from the related cycle $B C B C A C$ by applying the cyclic permutation $(A C B)$; moreover, this second cycle is constructed from a standard composition (1, 2, 3). For purposes of enumeration, therefore, it is necessary to consider only those cycles constructed from standard compositions. Calculations of optimal cost and cycle time for a cycle. from a non-standard composition may be performed by simple permutation of cost and demand parameters on the results for the corresponding standard form.

What constitutes "standard form"? It is clear that any cycle may be written with an $A$ in the first place: the cycle $B C B C A C$ above is equivalent to $A C B C B C$, obtained by restarting five places in. This leads to the concept of a mode, defined basically as a cycle constructed from a standard composition, beginning with an $A$, which cannot be obtained from any other mode by permutation and/or restarting. Thus $A C B C B C$ is the (only) mode from the standard composition (1, 2, 3).

If $n_{A} \geq 2$, rules are needed to standardize the starting point. These involve the lengths $\alpha_{i}$ of the spacings between successive $A$ 's; for $A B A C B C A C B, \alpha_{1}=1, \alpha_{2}=3, \alpha_{3}=2$.

(a) If there is a unique smallest $\alpha_{i}$, the mode places this first; thus $A B C A C B C B$ is the mode, not $A C B C B A B C$.

(b) If the smallest $\alpha_{i}$ is repeated, the mode has these least $\alpha_{i}$ as close as possible to the front of the cycle, beginning with one of them.

(c) If necessary, further ambiguity is resolved by taking the next smallest $\alpha_{i}$ as close as possible to the front; $A C A C B A C B A C A B C B C B$ is the mode with spacings $\alpha_{i}$ equal to $1,2,2,1,5$, and not $A C A B C B C B A C A C B A C B$, with spacings $1,5,1,2,2$. However, the smallest spacings between successive $A$ 's (two of length 1 ) occur in the same positions in both expressions here.

(d) Additional ambiguity may arise, even when the order of the spacings between the $A^{\prime}$ 's has been determined, if $n_{B}=n_{C}$. Where 
necessary, this is resolved by preferring $B$ to $C$ as early as possible in the cycle: $A B C B C$ is the (only) mode from $(1,2,2)$, not $A C B C B$.

Of course these rules have no intrinsic significance, and are used merely to allow standardized mode enumeration. We note in passing that repeated application of rule (c) may be necessary for very long, complex cycles.

\subsection{MODE NUMBER}

In the cycle-counting problem of this paper, enumeration of modes is clearly the cornerstone, underlying all that follows. A standard composition will in general have several associated modes. The mode number $F$ for a standard composition is the number of such modes. Thus for $(1,1,2), F=1$ (the mode is $A C B C$ ); for $(2,3,3$ ), $F=4$ (the modes are $A B A C B C B C, A B C A B C B C, A B C A C B C B, A B C B A C B C$ ). Finding the mode number for a given composition is far from trivial, and is the central theme of the succeeding sections.

\subsection{PERMUTATION NUMBER}

Each mode represents a collection of related permuted cycles, each having its own optimal time and cost. In general, the permutation number $G$ for a mode, or the number of distinct permuted cycles, is 6 . Degeneracy occurs, and is indicated wherever necessary in the mode discussion. For example, for the mode $A C B C, G=3$ ( $A C B C, B A C A, C B A B$ ); other cycles are restarts of these.

\subsection{MODUS OPERANDI}

The foregoing paragraphs indicate a suitable line of attack on our basic problem: "How many different cycles are there of given length $P$ ?" Firstly, identify standard compositions giving cycles of length $P$. Secondly, deduce the mode number for each, and count the total number of modes. Thirdly, count the associated cycles, taking degeneracies into account. The first theme is developed in section 3.

\section{Families of compositions}

Several useful comments, some already implied, may be made about the structure of cycles of three products. Firstly, immediate repetitions of letters need not be considered. Such a repetition implies slack time, 
since the machine lies idle while the inventory accumulated during the first run is consumed. Moreover, no set-up cost is involved for the second run, so that "immediate repetition" may be discarded in favour of "effective use of slack time".

Since sequences such as $A B B C$ or $A B A C A$ are not valid, it is clear that

$$
n_{i} \leq \frac{1}{2} P, \quad i \in\{A, B, C\}
$$

for all repetition numbers, and hence, since $n_{A}+n_{B}+n_{C}=P$, the set of $n_{i}$ satisfies the triangle law.

Consider the spaces of lengths $\alpha_{i}$ between successive $A^{\prime} s . B$ and $C$ must alternate in the available places, and each such space is characterized by its leading member $B$ or $C$. If $\alpha_{i}$ is odd, the leading letter appears once more in the space than the other. The multiplicity (mode and permutation) properties of a cycle in which such a sequence of $B^{\prime} s$ and $C^{\prime}$ s appears depend only on the parity of $\alpha_{i}$ and its leading member.

A principal composition is a standard composition in which $n_{A}=n_{B}$. Clearly $n_{A} \leq n_{C} \leq 2 n_{A}$ from the preceding remarks.

A family is a set of standard compositions characterized by a principal composition $\left(n_{A}, n_{B_{0}}, n_{C_{0}}\right)$ and an index $m$ : $\left[n_{A}, n_{B_{0}}+m, n_{C_{0}}+m\right]$. Thus $[1,1+m, 2+m]$ is the family with principal member $(1,1,2)$, followed by $(1,2,3),(1,3,4)$ and so on. Several properties may be mentioned.

(i) Every standard composition is a member of just one family. Consider any standard composition $\left(n_{A}, n_{B}, n_{C}\right)$, and define $k=n_{B}-n_{A}$, $\begin{aligned} n_{B_{0}}=n_{B}-k=n_{A}, & n_{C_{0}}=n_{C}-k . \text { Then } \\ & \left(n_{A}, n_{B}, n_{C}\right)=\left(n_{A}, n_{B_{0}}+k, n_{C_{0}}+k\right)\end{aligned}$

and $n_{B_{0}}, n_{C_{0}}, k$ are unique. Trivially, no principal composition can be 
a member of another family.

(ii) There are $n_{A}+1$ families involving a given $n_{A}$, since all $n_{C_{0}}$ between $n_{A}$ and $2 n_{A}$ inclusive are available.

(iii) Cycles constructed from all members of one family are either all even length or all odd length, since $n_{A}+n_{B_{0}}+n_{C_{0}}=P_{0}$ has the same parity as $P=P_{0}+2 m$. Hence a family is defined to be an even or odd family depending on the parity of its principal length $P_{0}$.

(iv) Consider a given cycle length $P$. Then every family of the same parity as $P$, with $P_{0} \leq P$, has just one member from which cycles of length $P$ are constructed; the index, $m$, of the nember is $\frac{z}{2}\left(P-P_{0}\right)$.

How many families have principal length $P$ ? Consider the principal member of such a family, $\left(n_{A}, n_{A}, n_{C_{0}}\right)$. Clearly $2 n_{A}+n_{C_{0}}=P$, $n_{C_{0}} \geq n_{A}, n_{C_{0}} \leq 2 n_{A}$. Hence $n_{A}, n_{A}, n_{C_{0}}$ must be a partition of $P$ with one repeated integer, and

$$
\begin{aligned}
& 3 n_{A} \leq P, \\
& 4 n_{A} \geq P .
\end{aligned}
$$

Any $n_{A}$ which satisfies

$$
\left\lceil\frac{1}{4} P\right\rceil \leq n_{A} \leq\left\lfloor\frac{1}{3} P\right\rfloor
$$

will generate a principal composition of length $P$. The number of these $n_{A}$ 's depends on the residue of $P$ modulo 12 . Suppose $P=12 k+a$. Then, for example, if $a=0$, there are $k+1$ of these $n_{A}$; if $a=1$, there are $k$ of them, and so on. In fact there are $k+1$ for all $a$ except 1,2 and 5 , for which there are $k$. These results are not difficult to derive.

How many families contribute a composition of given length $P$ ? (That is, how many families need be considered when discussing the number of modes of given length?) Clearly, all families of the same parity of $P$ 
for which $P_{0} \leq P$ contribute a composition. In the range $12 i$ to $12 i+11$, there are $6 i+4$ odd principal lengths and $6 i+5$ even ones, except that when $i=0$, there are only 4 (not 5 ) even ones. Suppose again that $P=12 k+a, a$ odd. Then there are

$$
\sum_{i=0}^{k-1}(6 i+4)=k(3 k+1)
$$

odd families with principal lengths less than or equal to $12 k-1$ and $k$ more if $a=1,2 k+1$ more if $a=3$, or $\frac{1}{2}(k(a+1)+a-3)$ more if $a>3$. If $a$ is even, there are

$$
\sum_{i=0}^{k-1}(6 i+5)-1=k(3 k+2)-1
$$

even families with principal lengths less than or equal to $12 k-2$, and $k+1$ more if $a=0,2 k+1$ more if $a=2$, or $\frac{1}{2}(k(a+2)+a)$ if $a>2$. A table may be constructed.

TABLE 3

Number of families contributing to given composition length

\begin{tabular}{l|c}
\multicolumn{1}{c|}{$P$} & No. of contributing families \\
\hline $12 k$ & $k(3 k+3)$ \\
$12 k+1$ & $k(3 k+2)$ \\
$12 k+2$ & $k(3 k+4)$ \\
$12 k+3$ & $k(3 k+3)+1$ \\
$12 k+4$ & $k(3 k+5)+1$ \\
$12 k+5$ & $k(3 k+4)+1$ \\
$12 k+6$ & $k(3 k+6)+2$ \\
$12 k+7$ & $k(3 k+5)+2$ \\
$12 k+8$ & $k(3 k+7)+3$ \\
$12 k+9$ & $k(3 k+6)+3$ \\
$12 k+10$ & $k(3 k+8)+4$ \\
$12 k+11$ & $k(3 k+7)+4$ \\
\hline
\end{tabular}

Thus, for example, when $P=27$ there are 19 contributing families, that is, 19 standard compositions giving rise to cycles of length 27 . The next problem to be faced is that of finding the number of modes available from each composition. Why consider families at all? Apart from 
making compositions simple to categorize and count, it is possible (at least for small $n_{A}$ ) to produce a general result giving the mode number for alz members of a family.

\section{Families with $n_{A}=1$}

As property (ii) of the previous section indicates, we have precisely two families with $n_{A}=1:[1,1+m, 1+m]$ and $[1,1+m, 2+m]$. These account for all standard compositions with $n_{A}=1$.

The family $[1,1+m, 1+m]$ has the single mode $A B C B C \ldots B C$, consisting of $A$ followed by $B C$ repeated $1+m$ times. Since interchange of $B$ and $C$ in this mode yields $A C B C B \ldots C B$, this is not considered to be a different mode. If $m>0$, the permutation number of the mode is 6 , because each of the six permutations on the set $\{A, B, C\}$ gives rise to a new cycle, while if $m=0$, composition $(1,1,1)$ has the single mode $A B C$ with permutation number 2 .

The family $[1,1+m, 2+m]$ also has only one mode, $A C B \ldots B C$, consisting of $A C$ followed by $B C$ repeated $1+m$ times. This has permutation number 6 if $m>0$, while if $m=0$ the permutation number is 3 :

\begin{tabular}{|c|c|c|}
\hline Fermutation & Cycle & \\
\hline $\begin{array}{l}(A)(B)(C) \\
(A B)(C)\end{array}$ & $\begin{array}{l}A C B C \\
B C A C\end{array}$ & same cycle \\
\hline $\begin{array}{l}(A C)(B) \\
(A B C)\end{array}$ & $\begin{array}{l}C A B A \\
B A C A\end{array}$ & same cycle \\
\hline $\begin{array}{l}(A)(B C) \\
(A C B)\end{array}$ & $\begin{array}{l}A B C B \\
C B A B\end{array}$ & same cycle \\
\hline
\end{tabular}

We summarise in a table. 
TABLE 4

Values of mode number, $F$, for $n_{A}=1$

\begin{tabular}{c|c|c|c|c|c}
$n_{B}{ }^{n} C$ & 1 & 2 & 3 & 4 & $\cdots$ \\
\hline 1 & 1 & 1 & & & \\
\hline 2 & & 1 & 1 & & \\
\hline 3 & & & 1 & 1 & \\
\hline $4 \cdot$ & & & & 1 & 1 \\
\hline$\vdots$ & & & & & 1. \\
\hline
\end{tabular}

Permutation number, $G$, is 6 , except in the cases of the modes for $(1,1,1)$ and $(1,1,2)$, which have $G=2$ and $G=3$ respectively.

\section{Families with $n_{A}=2$}

In this case there are 3 distinct families, which account for all the standard compositions with $n_{A}=2$.

\subsection{THE FamiLY $[2,2+m, 2+m]$}

Any mode is of the form $A \underset{\alpha_{1}}{\ldots} \underbrace{\ldots}_{\alpha_{2}}$, where the "gaps" between the

$A^{\prime}$ s are filled with $B^{\prime} s$ and $C^{\prime} s$, each $2+m$ times. Clearly any even gap must be filled either with $B C$ repeated a number of times, or with $C B$ repeated a number of times, while any odd gap is completed with either $C B \ldots B C$ (using one more $C$ than $B$ ) or $B C \ldots C B$ (using one more $B$ than $C$ ).

If $\alpha_{1}$ and $\alpha_{2}$ are odd, since $n_{B}=n_{C}$ and recalling the rules (a) and (d) in Section 2.2, we have $\alpha_{1} \leq \alpha_{2}$, and the $\alpha_{1}$-gap contains $B C \ldots C B$ while the $\alpha_{2}$-gap contains $C B \ldots B C$. Thus in this case there is the single mode $A \underbrace{B C \ldots C B}_{\alpha_{1}} A \underbrace{C B \ldots C}_{\alpha_{2}}$. 
If $\alpha_{1}$ and $\alpha_{2}$ are even, when $m>0$ and $\alpha_{1}<\alpha_{2}$ there are two modes :

$$
A \underbrace{B C \ldots B C}_{\alpha_{1}} A \underbrace{B C \ldots B C}_{\alpha_{2}} \text { and } A \underbrace{B C \ldots B C}_{\alpha_{1}} A \underbrace{C B \ldots C B}_{\alpha_{2}} \text {. }
$$

When $\alpha_{1}=\alpha_{2}=2+m$ is even and $m>0$, there is a single mode:

$$
A \underbrace{B C \ldots B C A C B \ldots C B}_{2+m} \text {. }
$$

This is because the cycle $A \underbrace{B C \ldots B C}_{2+m} A \underbrace{B C \ldots B C}_{2+m}$ is not counted, being two copies of the cycle $A B C \ldots B C$ of length $3+m$, arising from the standard composition $\left(1, \frac{1}{2}(2+m), \frac{1}{2}(2+m)\right)$.

When $m=0$, we have the case of the principal composition $(2,2,2)$, which has the single mode $A B A C B C$, since the cycle $A B C A C B$ with the permutation $(A B C)$ applied to it produces the cycle $B C A B A C$, which is the mode $A B A C B C$ once again.

Now the integer $4+2 m$ partitions into two positive parts thus:

$$
\begin{aligned}
4+2 m= & 1+(2 m+3) \\
2+(2 m+2) & \\
& \cdots \\
& (m+2)+(m+2)
\end{aligned}
$$

It follows that the mode number, $F$, is

$$
\begin{aligned}
& \frac{1}{2}(m+3)+2 \cdot \frac{1}{2}(m+1) \text { if } m \text { is odd, } \\
& \frac{1}{2}(m+2)+2 \cdot \frac{1}{2} m+1 \text { if } m \text { is even, } m>0 .
\end{aligned}
$$

That is,

$$
F= \begin{cases}\frac{1}{2}(3 m+5), & m \text { odd }, \\ \frac{1}{2}(3 m+4), & m \text { even, } m>0, \\ 1 & , m=0 .\end{cases}
$$

Permutation numbers, $G$, are 6 , except in the following cases:

the mode $A B A C B C$ has $G=3$;

the modes $A \underbrace{B C \ldots B C}_{m+2} A \underbrace{C B \ldots C B}_{m+2}$ ( $m$ even, $m>0$ ) have $G=3$; 
the modes $A \underbrace{B C \ldots C B}_{m+2} A \underbrace{C B \ldots B C}_{m+2}$ ( $m$ odd) have $G=3$.

\subsection{THE FAMILY $[2,2+m, 3+m]$}

As in the previous section, the value of $F$ in the case $m=0$ (the principal composition for this family) is lower than might be expected. The composition $(2,2,3)$ has modes

\section{$A C A B C B C$ and $A B C A C B C$,}

so $F=2$ here. (The cycle $A C A C B C B$ is equivalent to the first of these two modes under the permutation $(A B)(C)$, while the cycle $A C B A C B C$ is equivalent to the second mode, also under $(A B)(C)$.) Each of these two modes has permutation number 6 .

When $m>0, n_{A}, n_{B}$ and $n_{C}$ are all distinct, so all modes in this family will have permutation number 6 . Consider the mode $A \underbrace{\ldots}_{\alpha_{1}} \underbrace{\ldots}_{\alpha_{2}}$, where $\alpha_{1}+\alpha_{2}=5+2 m$, which is odd. Thus one of $\alpha_{1}, \alpha_{2}$ must be odd and the other one even. Since $n_{C}=n_{B}+1$, the odd "gap" must be filled with $C B \ldots B C$, while the even "gap" may be completed with either $B C \ldots B C$ or $C B \ldots C B$. So for each partition of $5+2 m$ into two positive parts, we have two modes. Now

$$
\begin{aligned}
& 5+2 m= 1+(2 m+4) \\
& 2+(2 m+3) \\
& \\
& \quad \cdots \\
&(m+2)+(m+3) .
\end{aligned}
$$

Thus for any $m>0, F=4+2 m$ for this family, while $F=2$ when $m=0$.

\subsection{THE FAMILY $[2,2+m, 4+m]$}

In this case the spaces $\alpha_{1}$ and $\alpha_{2}$ between the $A^{\prime}$ 's must both be odd and completed with $C B \ldots B C$, since $n_{C}=n_{B}+2$. The case $m=0$, $(2,2,4)$, has the single mode

\section{$A C A C B C B C$,}

which has permutation number 3 , because the cycle $A C B C A C B C$ is really twice the cycle $A C B C$ of length $P=4$ instead of $P=8$. 
When $m>0$, we partition $6+2 m$ into two odd parts:

$$
\begin{aligned}
6+2 m= & 1+(2 m+5), \\
3+(2 m+3), & \cdots \\
& \left\{\begin{array}{l}
(m+3)+(m+3) \text { if } m \text { is even, } \\
(m+2)+(m+4) \text { if } m \text { is odd. }
\end{array}\right.
\end{aligned}
$$

However the partition $6+2 m=(m+3)+(m+3)$ when $m$ is even contributes no modes to the count of $F$, because

$$
A \underbrace{C B \ldots B C}_{m+3} A \underbrace{C B \ldots B C}_{m+3}
$$

is two copies of the mode $\underbrace{C B \ldots B C}_{m+3}$ of half the length. Thus we have

$$
F= \begin{cases}\frac{1}{2}(m+2) & \text { if } m \text { is even, } m \geq 0, \\ \frac{1}{2}(m+3) & \text { if } m \text { is odd. }\end{cases}
$$

Each mode for $m>0$ has permutation number $G=6$.

5.4. SUMMARY FOR $n_{A}=2$

TABLE 5.4

Values of mode number $F$ for $n_{A}=2$

\begin{tabular}{|c|c|c|c|c|c|c|c|c|c|c|}
$n_{B}{ }^{n_{C}}$ & 2 & 3 & 4 & 5 & 6 & 7 & 8 & 9 & 10 & 11 \\
\hline 2 & 1 & 2 & 1 & & & & & & & \\
\hline 3 & & 4 & 6 & 2 & & & & & & \\
\hline 4 & & & 5 & 8 & 2 & & & & & \\
\hline 5 & & & & 7 & 10 & 3 & & & & \\
\hline 6 & & & & & 8 & 12 & 3 & & & \\
\hline 7 & & & & & & 10 & 14 & 4 & & \\
\hline 8 & & & & & & & 11 & 16 & 4 & \\
\hline 9 & & & & & & & & 13 & 18 & 5 \\
\hline 10 & & & & & & & & & 14 & 20 \\
\hline
\end{tabular}


In Table 5.4, permutation number, $G$, is 6 , except for the modes for the principal compositions $(2,2,2)$ and $(2,2,4)$, which have $G=3$, and one mode for each of the compositions in the family $[2,2+m, 2+m]$, which also have $G=3$.

\section{Families with $n_{A}=3$}

\section{1 .}

of the four families $[3,3+m, 3+m],[3,3+m, 4+m],[3,3+m, 5+m]$ and $[3,3+m, 6+m]$, we shall deal in detail with the first, and merely state results for the remaining three. In all cases, our general mode is of the form

$$
A \underbrace{\ldots}_{\alpha_{1}} A \underbrace{\ldots}_{\alpha_{2}} \underbrace{\ldots}_{\alpha_{3}}
$$

where $\alpha_{1}+\alpha_{2}+\alpha_{3}=n_{B}+n_{C}$. Thus we are interested in partitions of $n_{B}+n_{C}$ into three non-zero parts. It is straightforward to show, using generating functions, that the number of partitions of $n$ into three nonzero parts, $p_{3}(n)$, is:

$$
\begin{aligned}
& \frac{1}{12} n^{2} \text { if } n \equiv 0(\bmod 6) ; \\
& \frac{1}{12}\left(n^{2}-1\right) \text { if } n \equiv 1,5(\bmod 6) ; \\
& \frac{1}{12}\left(n^{2}-4\right) \text { if } n \equiv 2,4(\bmod 6) ; \\
& \frac{1}{12}\left(n^{2}+3\right) \text { if } n \equiv 3(\bmod 6) .
\end{aligned}
$$

However we do not use this result until the next section, because in practice to find the number of modes for standard compositions with $n_{A}=3$, more detail is required about the partitions: whether the $\alpha_{i}$ are all distinct or two are equal, how many are odd, and so on.

\subsection{THE FAMILY $[3,3+m, 3+m]$}

Consider partitions of $6+2 m$ into $\alpha_{1}+\alpha_{2}+\alpha_{3}$. Either all three parts will be even, or two will be odd and one even. When $\alpha_{1}, \alpha_{2}$ and $\alpha_{3}$ are even and distinct, there are four modes that may be constructed: 


$$
\begin{aligned}
& A \underbrace{B C \ldots B C}_{\alpha_{1}} A \underbrace{B C \ldots B C}_{\alpha_{2}} A \underbrace{B C \ldots B C}_{\alpha_{3}} \text {; } \\
& A \underbrace{B C \ldots B C}_{\alpha_{1}} A \underbrace{B C \ldots B C}_{\alpha_{2}} A \underbrace{C B \ldots C B}_{\alpha_{3}} \text {; } \\
& A \underbrace{B C \ldots B C}_{\alpha_{1}} A \underbrace{C B \ldots C B}_{\alpha_{2}} A \underbrace{B C \ldots B C}_{\alpha_{3}} \text {; } \\
& A \underbrace{B C \ldots B C}_{\alpha_{1}} A \underbrace{C B \ldots C B}_{\alpha_{2}} A \underbrace{C B \ldots C B}_{\alpha_{3}} \text {. }
\end{aligned}
$$

Similarly, taking the gaps between $A^{\prime} s$ in the order $\alpha_{1}, \alpha_{3}, \alpha_{2}$ gives rise to another four such modes. Moreover, if $\alpha_{1}=\alpha_{2} \neq \alpha_{3}$ and $\alpha_{1}, \alpha_{2}$, $\alpha_{3}$ are all even, we have four modes, and if $\alpha_{1}=\alpha_{2}=\alpha_{3}$, even, we have only one mode.

When two of the $\alpha_{i}$ are odd and the third is even, we have modes of the form (with $\alpha_{1}$ even):

$$
A \underbrace{B C \ldots B C}_{\alpha_{1}} A \underbrace{B C \ldots C B}_{\alpha_{2}} A \underbrace{C B \ldots B C}_{\alpha_{3}}
$$

or

$$
A \underbrace{B C \ldots B C}_{\alpha_{1}} A \underbrace{C B \ldots B C}_{\alpha_{2}} A \underbrace{B C \ldots C B}_{\alpha_{3}} .
$$

If the two odd numbers are distinct we obtain two more such modes by re-ordering the partition: two from $\alpha_{1}, \alpha_{2}, \alpha_{3}$ and two from $\alpha_{1}, \alpha_{3}, \alpha_{2}$.

The following result was found by ad hoc methods but is most easily verified by induction on $m$.

PROPOSITION 6.2.1. The family $[3,3+m, 3+m]$ has the following mode number:

$$
\begin{array}{ll}
\frac{1}{3}\left(5 m^{2}+21 m+21\right) & \text { when } m \equiv 0(\bmod 3), m>0 ; \\
\frac{1}{3}\left(5 m^{2}+21 m+22\right) & \text { when } m \equiv 1,2(\bmod 3) ; \\
3 & \text { when } m=0 .
\end{array}
$$

Proof. We use induction on $m$, in steps of size 3 . The case 
$m=0$ fails to fit the "pattern"; it has modes

$A B A C A C B C B$, with permutation number 2 ,
$A B A B C A C B C$, with permutation number 6 ,
$A B A C B C A B C$, with permutation number 6 .

The cases $m=1,2,-3$ begin the induction.

Case $m=1,(3,4,4)$. Partitions of 8 are : $1,1,6$; $1,2,5 ; 1,3,4 ; 2,2,4 ; 2,3,3$. Thus we have

$$
F=2+4+4+4+2=16=\frac{1}{3}(5+21+22) \text {. }
$$

Case $m=2,(3,5,5)$. Partitions of 10 are: $1,1,8$; $1,2,7 ; 1,3,6 ; 1,4,5 ; 2,2,6 ; 2,3,5 ; 2,4,4 ; 3,3,4$. Thus

$$
F=2+4+4+4+4+4+4+2=28=\frac{1}{3}(5 \cdot 4+21 \cdot 2+22) \text {. }
$$

Case $m=3,(3,6,6)$. In the same manner, using partitions of 12 , we obtain $F=43$ (noting that the partition $4+4+4$ yields mode number 1 ), and $43=\frac{1}{3}(5 \cdot 9+21.3+21)$, as required.

Now consider the inductive step. Increasing $m$ by 3 increases $n_{B}+n_{C}$ by 6 , from $6+2 m$ to $12+2 m$. Partitions of $12+2 m$ into three parts, each of size at least 3 , arise from all partitions of $6+2 m$ into three parts by adding 2 to each of the three partition members. Thus we need only consider partitions $12+2 m=\alpha_{1}+\alpha_{2}+\alpha_{3}$ where $\alpha_{1}=1$ or $\alpha_{1}=2$. These are:

$$
\begin{array}{cc}
1+1+(2 m+10) ; & 2+2+(2 m+8) ; \\
1+2+(2 m+9) ; & 2+3+(2 m+7) ; \\
\cdots & \cdots \\
1+(m+5)+(m+6) ; & 2+(m+5)+(m+5)
\end{array}
$$

If $m$ is even, these partitions contribute the following to $F$ :

$$
(2+4(m+4))+\left(\frac{1}{2}(m+2) \cdot 4+2\right)+\left(4+\frac{1}{2}(m+2) \cdot 8\right)=10 m+36 .
$$

Similarly if $m$ is odd, they contribute the following to $F$ :

$$
(2+4(m+4))+\left(\frac{1}{2}(m+3) \cdot 4\right)+\left(4+4+\frac{1}{2}(m+1) \cdot 8\right)=10 m+36 .
$$

Now by the inductive hypothesis, when $m \equiv 0(\bmod 3), m>0$, the family $[3,3+(m+3), 3+(m+3)]$ has $F$ equal to 


$$
\frac{1}{3}\left(5 m^{2}+21 m+21\right)+10 m+36=\frac{1}{3}\left(5(m+3)^{2}+21(m+3)+21\right),
$$

and when $m \equiv 1$ or $2(\bmod 3)$, it has $F$ equal to

$$
\frac{1}{3}\left(5 m^{2}+21 m+22\right)+10 m+36=\frac{1}{3}\left(5(m+3)^{2}+21(m+3)+22\right) .
$$

The result follows.

6.2.2 PERMUTATION NUMBERS. The case $m=0$ has already been dealt with above. In all other cases, only the permutation $(A)(B C)$ which interchanges $B$ and $C$ could give rise to a mode which has already occurred, since $n_{B}=n_{C}>n_{A}$. However, inspection of the mode types shows that they all have $G=6$.

6.3. THE REMAINING FAMILIES WITH $n_{A}=3$

We state the following results without proof; they may all be proved by induction along the lines of the proof of Proposition 6.2.1.

PROPOSITION 6.3.1. (i) The formily $[3 ; 3+m, 4+m]$ has mode number

$$
\begin{array}{ll}
\frac{1}{2}(m+3)(5 m+12) & \text { if } m>0, \\
10 & \text { if } m=0 .
\end{array}
$$

(ii) AZZ permutation numbers for the above modes are 6 , except for the modes

$$
A C A C A B C B C B \text { and } A B C A C B A C B C \text {, }
$$

in the case $m=0$; these each have permutation number 3 .

PROPOSITION 6.3.2. (i) The farily $[3,3+m, 5+m]$ has mode number

$$
\begin{array}{ll}
m^{2}+7 m+12 \text { if } m>0, \\
6 & \text { if } m=0 .
\end{array}
$$

(ii) AZZ permutation numbers for the above modes are 6 .

PROPOSITION 6.3.3. (i) The famizy $[3,3+m, 6+m]$ has mode number

$$
\begin{array}{ll}
\frac{1}{5}(m+4)(m+5) & \text { if } m \neq 0(\bmod 3), \\
\frac{1}{6}(m+3)(m+6) & \text { if } m \equiv 0(\bmod 3), m>0, \\
2 & \text { if } m=0 .
\end{array}
$$

(ii) All permutation numbers for the above modes are 6 , except for the following mode in the case $m=0$, which has $G=3$ : 


\section{$A C A C A C B C B C B C$.}

TABLE 6.4

Values of $F$ when $n_{A}=3$

\begin{tabular}{|c|c|c|c|c|c|c|c|c|c|c|}
\hline I & 3 & 4 & 5 & 6 & 7 & 8 & 9 & 10 & 11 & 12 \\
\hline 3 & 3 & 10 & 6 & 2 & & & & & & \\
\hline 4 & & 16 & 34 & 20 & 5 & & & & & \\
\hline 5 & & & 28 & 55 & 30 & 7 & & & & \\
\hline 6 & & & & 43 & 81 & 42 & 9 & & & \\
\hline 7 & & & & & 62 & 112 & 56 & 12 & & \\
\hline 8 & & & & & & 84 & 148 & 72 & 15 & \\
\hline 9 & & & & & & & 109 & 189 & 90 & 18 \\
\hline 10 & & & & & & & & 138 & 235 & 110 \\
\hline 11 & & & & & & & & & 170 & 286 \\
\hline
\end{tabular}

\section{An estimate for general $n_{A}$}

\subsection{THE FORM OF THE ESTIMATE}

Although detailed discussion of cycles with $n_{A}>3$ is possible, the analysis will become very involved as $n_{A}$ increases. We now obtain an estimate, almost certainly an over-estimate, for the total number of modes with prescribed cycle length $P$ and number of $A^{\prime}$ 's, $n_{A}$.

First, we note that placement of the $n_{A} A^{\prime}$ 's, starting with an $A$, leaves $P-n_{A}$ positions to be occupied by $B^{\prime} s$ and $C^{\prime} s$. These $P-n_{A}$ positions are divided into $n_{A}$ pieces of lengths $\alpha_{1}, \alpha_{2}, \ldots, \alpha_{n_{A}}$, which appear in some order. As mentioned in Section 6 , the $\alpha_{i}$ form a partition of $P-n_{A}$ into $n_{A}$ pieces; there are $p_{n_{A}}\left(P-n_{A}\right)$ such partitions.

Next, these pieces can be arranged in at most $\left(n_{A}-1\right) !$ ways, 
remembering that we always begin with the least $\alpha_{i}$, or a specified least one (see Section 2.2). Some of these $\left(n_{A}-1\right)$ ! arrangements will not produce distinct modes. Reshuffling repeated $\alpha_{i}$ will not give a different mode, while some arrangements will violate requirement (b) or (c) of Section 2.2. Hence the approximation $p_{n_{A}}\left(P-n_{A}\right) \cdot\left(n_{A}-1\right) !$ as the number of mode-generating placements of the $n_{A} A^{\prime} \mathrm{s}$ is an overestimate of the actual number.

Next, let us consider the number of ways that $B^{\prime} s$ and $C^{\prime} s$ can occupy the remaining positions, subject always to the proviso $n_{B} \leq n_{C}$. Only the leading letter of a piece of length $\alpha_{i}$ need be considered. Suppose a partition $\alpha_{1}, \alpha_{2}, \ldots, \alpha_{n_{A}}$ consists of $\lambda$ odd numbers and $\mu$ even numbers, so $\lambda+\mu=n_{A}$. Discussion in earlier sections shows that any even length piece may begin with $B$ or $C$, and up to half the odd length pieces may begin with $B$. Two cases arise.

(a) $\lambda$ is odd. $B$ may be the first letter in $0,1, \ldots$, or $(\lambda-1) / 2$ of the odd pieces. Thus $B$ may begin 0 of these in 1 way, 1 of these in $\left(\begin{array}{l}\lambda \\ 1\end{array}\right)$ ways, and so on, with a total number for the odd pieces of $\sum_{i=0}^{\frac{1}{2}(\lambda-1)}\left(\begin{array}{l}\lambda \\ i\end{array}\right)$. Moreover, the number of ways of beginning the even pieces is $2^{\mu}$. Thus the total number of ways of inserting $B^{\prime} s$ and $C^{\prime}$ s in this case is

$$
\begin{aligned}
2^{\mu} \sum_{i=0}^{\frac{1}{2}(\lambda-1)}\left(\begin{array}{l}
\lambda \\
i
\end{array}\right) & =2^{\mu} \cdot \frac{1}{2} \cdot \sum_{i=0}^{\lambda}\left(\begin{array}{l}
\lambda \\
i
\end{array}\right) \text {, since } \lambda \text { is odd, } \\
& =2^{\mu+\lambda-1} \\
& =2^{n} A^{-1} .
\end{aligned}
$$

(b) $\lambda$ is even. A similar argument leads to a number of ways of inserting $B^{\prime} s$ and $C^{\prime}$ s equal to 


$$
\begin{aligned}
2^{\mu} \sum_{i=0}^{\frac{1}{2} \lambda}\left(\begin{array}{l}
\lambda \\
i
\end{array}\right) & =2^{\mu-1}\left[\sum_{i=0}^{\lambda}\left(\begin{array}{l}
\lambda \\
i
\end{array}\right)+\left(\begin{array}{c}
\lambda \\
\frac{1}{2} \lambda
\end{array}\right)\right] \\
& =2^{\mu-1}\left[2^{\lambda}+\left(\begin{array}{c}
\lambda \\
\frac{1}{2} \lambda
\end{array}\right)\right] \\
& =2^{\lambda+\mu-1}\left[1+2^{-\lambda}\left(\begin{array}{c}
\lambda \\
\frac{1}{2} \lambda
\end{array}\right)\right] .
\end{aligned}
$$

For this case, the approximation $2^{n^{-1}}$ as the number of ways of placing $B^{\prime} s$ and $C^{\prime} s$ is an under-estimate, although the proportional error decreases quickly as $\lambda$ increases.

Finally, the estimate for the number of modes with prescribed $P$ and $n_{A}$ is

$$
E\left(P, n_{A}\right)=2^{n_{A}-1}\left(n_{A}-1\right) ! p_{n_{A}}\left(P-n_{A}\right)
$$

Although it is not completely clear whether this is an over or underestimate, we conjecture that the possibilities for duplication in placing of $A^{\prime} s$ outweigh the cases lost in the estimate for placing of $B^{\prime} s$ and $C^{\prime}$ 's, particularly for larger $n_{A}$. Further conjectures on the accuracy and usefulness of the estimate will be made after comparison with the actual figures for $n_{A}$ equal to 2 and 3 . Trivially, $E(P, 1)=1$, and this is exact, because only one mode exists when $n_{A}=1$.

\subsection{PERFORMANCE FOR $n_{A}=2$}

When $n_{A}=2$,

$$
p_{2}(P-2)=\left\{\begin{array}{lll}
\frac{1}{2}(P-3), & P & \text { odd } \\
\frac{1}{2}(P-2), & P & \text { even }
\end{array}\right.
$$

Thus

$$
E(P, 2)=\left\{\begin{array}{lll}
P-3, & P & \text { odd } \\
P-2, & P & \text { even }
\end{array}\right.
$$

The actual number of modes, say $A(P, 2)$, also depends on the parity of $P$. Suppose $P$ is even. Then the modes will be made up of contributions from the families $\left[2,2+m_{1}, 2+m_{1}\right], m_{1}=\frac{1}{2}(P-6)$, and $\left[2,2+m_{2}, 4+m_{2}\right]$, 
$m_{2}=\frac{1}{2}(P-8)$. Clearly $m_{1}$ and $m_{2}$ have opposite parities, and the results of Section 5 show that

$$
A(P, 2)=P-3,
$$

unless $P=6$, when $A(6,2)=1$; this corresponds to the exceptional case $m_{1}=0$.

If $P$ is odd, a similar argument shows that again $A(P, 2)=P-3$, unless $P=7$, when $A(7,2)=2$. Table 7.1 shows the comparison between actual and estimated values.

TABLE 7.1

Actual and estimated mode numbers for $n_{A}=2$, with estimation errors

\begin{tabular}{cccc}
$P$ & $A(P, 2)$ & $E(P, 2)$ & Error $\varepsilon$ \\
\hline 6 & 1 & 4 & 3 \\
7 & 2 & 4 & 2 \\
even $P$ & $P-3$ & $P-2$ & 1 \\
odd $P$ & $P-3$ & $P-3$ & 0
\end{tabular}

Several points are worth noting. Firstly, the estimate $E(P, 2)$ is linear in $P$, and the error $\varepsilon$ is constant; so the error in the estimate is one order lower in $P$ than the estimate. Thus the proportional error decreases as $P$ increases. Secondly, the estimate is excessive for values of $P$ such that early exceptional behaviour occurs in the contributing families. This exceptional behaviour arises from degeneracy; for these marginal values, here 6 and 7 , the estimate for a value of $n_{A}$ one smaller (namely $n_{A}=1$ ) is better. Thirdly, the estimate for $n_{A}=2$ is a reasonable one for the total number of all modes for $n_{A} \leq 2$. This is related to the first comment above. These points are amplified in the next section.

\subsection{PERFORMANCE FOR $n_{A}=3$}

Using the remark from Section 6 concerning $p_{3}(n)$, we obtain 


$$
E(P, 3)= \begin{cases}\frac{1}{3}\left(2 P^{2}-12 P+24\right), & P \equiv 0(\bmod 6), \\ \frac{1}{3}\left(2 P^{2}-12 P+18\right), & P \equiv 3(\bmod 6), \\ \frac{1}{3}\left(2 P^{2}-12 P+16\right), & P \equiv 2,4(\bmod 6), \\ \frac{1}{3}\left(2 P^{2}-12 P+10\right), & P \equiv 1,5(\bmod 6) .\end{cases}
$$

Combining the family results from Section 6 , by an argument similar to that above for $n_{A}=.2$, we obtain general results for $A(P, 3)$. Exceptional values occur when families make their first contributions. Table 7.2 (see p. 428) sumarises the results.

Again, there are several features of interest. The general estimate is quadratic in $P$ with a linear error, one order lower as before. $E(P, 3)$ provides a reasonable estimate for the sum of all actual values for $n_{A} \leq 3$. Bad proportional over-estimates occur for early values, where exceptional behaviour arises as families first make their contribution. Moreover, not all the families contribute until $P=12$, when the estimate takes the general form, and the error is extremely close to the general expression. It is worth noting also that the most severe degeneracy occurs for the most symmetrical family $[3,3+m, 3+m]$, when $P=9$ and $m=0$. As before, the one-smaller estimate $E(9,2)$ is better $(E(9,2)=6$ whereas $E(9,3)=24)$, since only a small contribution from families with $n_{A}=3$ has arisen at that stage.

\subsection{AN HEURISTIC IMPROVEMENT}

It appears that the estimate $E\left(P, n_{A}\right)$ is good provided that $n_{A}$ is such that new families are not involved. Particularly for the case where $P=3 n_{A}$, the estimate is much too great. In this case, earlier evidence suggests that the estimate using $n_{A}-1$ is much better; as $P$ increases to the level $3 n_{A}+n_{A}$, when all families are contributing (recall that the last family to contribute, $\left[n_{A}, n_{A}+m, 2 n_{A}+m\right]$, is only slightly degenerate for $m=0), E\left(P, n_{A}\right)$ is usable, and appears to give a reasonable estimate for the total number of modes with $n_{A}$ and fewer $A^{\prime} \mathrm{s}$. 


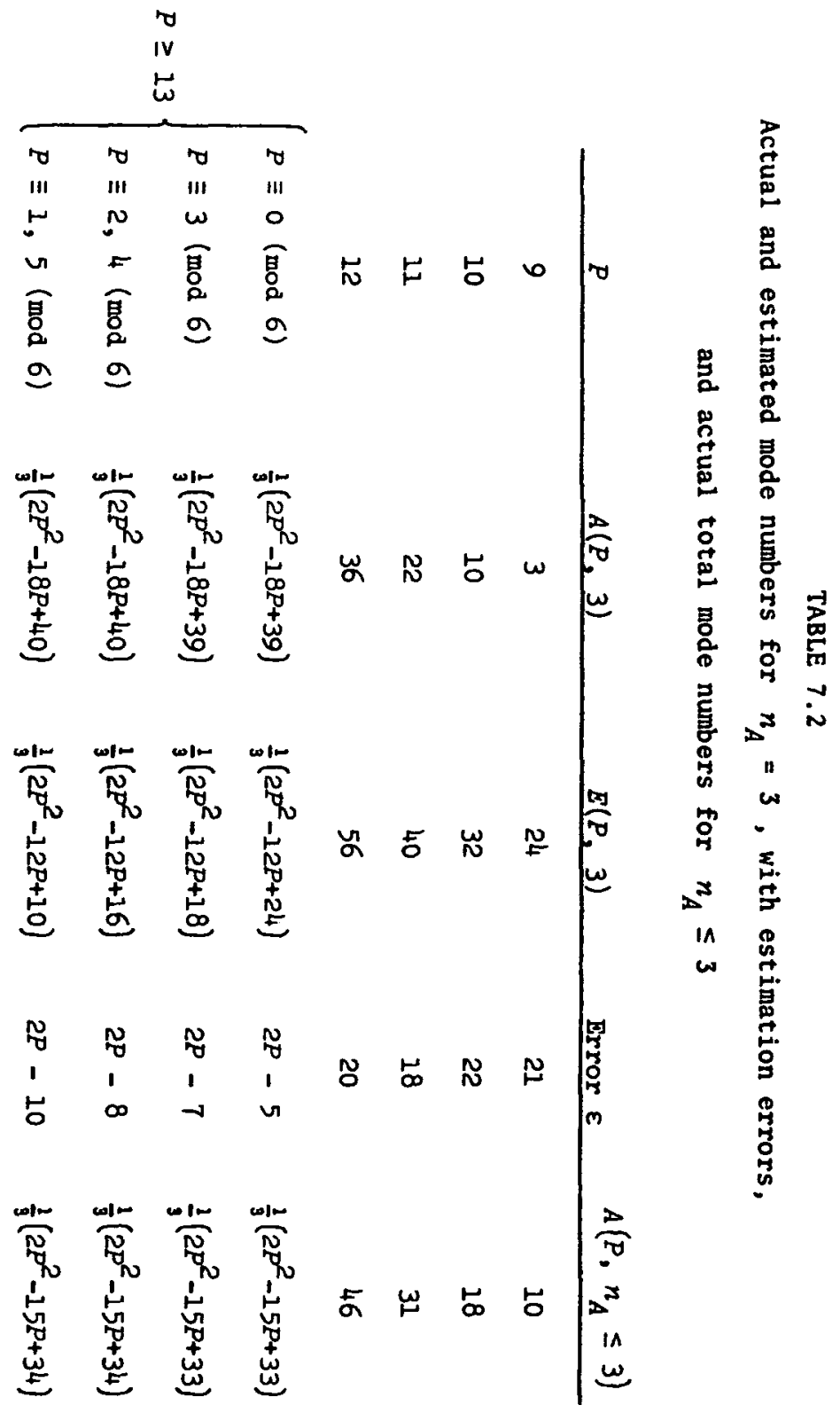


Various heuristic sliding scale schemes have been considered, to "phase in" use of $E\left(P, n_{A}\right)$ over $E\left(P, n_{A}-1\right)$, where $n_{A}$ is $\left\lfloor\frac{1}{3} P\right\rfloor$. Observations made in the cases $n_{A}=2$ and 3 indicate that a better estimate is obtained by involving $E\left(P, n_{A}\right)$ on a linear sliding scale. This "phasing in" gives reasonable results if $E\left(P, n_{A}\right)$ is not considered at all until $P=3 n_{A}+1$, and finally when $P=4 n_{A}$, the estimate taken is solely $E\left(P, n_{A}\right)$. For example, when $P=7$, $\frac{1}{2} E(7,1)+\frac{1}{2} E(7,2)=2.5$ gives a reasonable estimate, noting that $F=3$ when $P=7$. And when $P=10, \frac{2}{3} E(10,2)+\frac{1}{3} E(10,3)=16$, while $E=18$ when $P=10$. Other estimated values are given in Table 7.3, for $P$ from 3 to 12 . (The mode number for the composition $(4,4,4)$ can be shown to be 13. )

TABLE 7.3

Comparison between actual total number of modes and estimated number, using an heuristic sliding scale for the estimate

\begin{tabular}{rccrl}
$P$ & $\max n_{A}$ & $E$ & Estimate \\
\hline 3 & 1 & 1 & $E(3,1)=1$ \\
4 & 1 & 1 & $E(4,1)=1$ \\
$E(5,1)=1$ \\
5 & 1 & 1 & $1 . E(6,1)+0 . E(6,2)=1$ \\
6 & 2 & 2 & $\frac{1}{2} E(7,1)+\frac{1}{2} E(1,2)=2.5$ \\
1 & 2 & 3 & $0 . E(8,1)+1 . E(8,2)=6$ \\
8 & 2 & 6 & $1 . E(9,2)+0 . E(9,3)=6$ \\
9 & 3 & 10 & $\frac{2}{3} E(10,2)+\frac{1}{3} E(10,3)=16$ \\
10 & 3 & 18 & $\frac{1}{3} E(11,2)+\frac{2}{3} E(11,3)=29.3$ \\
11 & 3 & 31 & $0 . E(12,2)+1 . E(12,3)=56$ \\
12 & 4 & 59 &
\end{tabular}

\section{Practical considerations}

The above discussion highlights perhaps the most important contribution made by this paper: for three products, the number of cycles that need to be considered in an exhaustive search for optimal cost is much smaller than one might anticipate. A naive (and apprehensive!) estimator might suggest $3.2^{P-1}$ cycles of length $P$ exist, since any letter can be 
followed by either of the other two. Thus for $P=12$, he might estimate 6,144 cycles, compared with an actual figure of less than 6 times 59 (less than, since some permutations are degenerate).

Similarly, even for $P$ as high as 17 , our sliding heuristic estimate gives 2515 modes, and indeed fewer than 5000 modes, 30,000 cycles, for $P$ less than or equal to 17 , for all possible $n_{A}$ 's . Such a search would be well within the capabilities of even a modest computing facility. Even better, in practice one would have a good idea of the upper limit for $n_{A}$, given $P$, from information about the independent cycle solutions, limiting still further the number of calculations required to ensure a close to optimal result. We comment, finally, that the heuristic estimate has not been checked against sufficient actual values to justify its practical use, although we suspect that it is still a fairly high overestimate for larger $P$. Table 8 shows values of the estimated number of modes with cycle length $P, E^{*}(P)$, and $E_{\text {CUM }}^{*}(P)$, the total number of modes (rounded) for cycles of length less than or equal to $P$, using the estimate.

TABLE 8

Estimate, and cumulative estimate for cycle length up to 20

\begin{tabular}{lcr}
$P$ & $E^{*}(P)$ & $E_{\text {CUM }}^{*}(P)$ \\
\hline 13 & 120 & 240 \\
14 & 256 & 496 \\
15 & 420 & 916 \\
16 & 1344 & 2260 \\
17 & 2515.2 & 4775 \\
18 & 4358.4 & 9133 \\
19 & 15064 & 24197 \\
20 & 32000 & 56197 \\
\hline
\end{tabular}




\section{References}

[1] Earl E. Bomberger, "A dynamic programming approach to a lot size scheduling problem", Management Sci. Ser. A 12 (1966), 778-784.

[2] Salah E. Elmaghraby, "The economic lot scheduling problem (ELSP): review and extensions", Management Sci. 24 (1977/1978), 587-598.

[3] James M. Fitz-Gerald, "Repetitive multiple period cycles in the Economic Lot Scheduling problem" (Operations Research Report, University of Queensland, St Lucia, to appear).

\footnotetext{
Department of Mathematics,

University of Queensland,

St Lucia,

Queensland 4067,

Australia.
} 